\title{
On Conjugation Partitions of Sets of Trinucleotides
}

\author{
Lorenzo Bussoli ${ }^{1}$, Christian J. Michel $^{2}$, Giuseppe Pirillo ${ }^{1,3}$ \\ ${ }^{1}$ Dipartimento di Matematica U.Dini, Firenze, Italia \\ ${ }^{2}$ Equipe de Bioinformatique Théorique, Université de Strasbourg, \\ Strasbourg, France \\ ${ }^{3}$ Université de Marne-la-Vallée, Marne-la-Vallée, France \\ Email: \{bussoli, pirillo\}@math.unifi.it,michel@dpt-info.u-strasbg.fr
}

Received October 2, 2011; revised December 5, 2011; accepted December 13, 2011

\begin{abstract}
We prove that a trinucleotide circular code is self-complementary if and only if its two conjugated classes are complement of each other. Using only this proposition, we prove that if a circular code is self-complementary then either both its two conjugated classes are circular codes or none is a circular code.
\end{abstract}

Keywords: Trinucleotide; Conjugated Trinucleotides; Code; Circular Code; Self-Complementary Circular Code; Complementary Circular Codes

\section{Introduction}

We continue our study of the combinatorial properties of trinucleotide circular codes. A trinucleotide is a word of three letters (triletter) on the genetic alphabet $\{A, C, G, T\}$. The set of 64 trinucleotides is a code in the sense of language theory, more precisely a uniform code, but not a circular code [1,2]. In order to have an intuitive meaning of these notions, codes are written on a straight line while circular codes are written on a circle, but, in both cases, unique decipherability is required.

Comma free codes, a very particular case of circular codes, have been studied for a long time, e.g. [3-5]. After the discovery of a circular code in genes with important properties [6], circular codes are mathematical objects studied in combinatorics, theoretical computer science and theoretical biology, e.g. [7-23].

There are 528 self-complementary circular codes of 20 trinucleotides $[6,24,25]$ and, as proved here, they are naturally partitioned into two quite symmetric classes.

Let $\mathcal{T}=\{A A A, C C C, G G G, T T T\}$ be the four trinucleotides with identical nucleotides. In this paper, we study some particular partitions of $\mathcal{A}_{4}^{3} \backslash \mathcal{T}$. Indeed, each circular code $X_{0}$ can be associated with two other subsets $X_{1}$ and $X_{2}$ of $\mathcal{A}_{4}^{3} \backslash \mathcal{T}$ simply by operating two circular permutations of one letter and two letters on the trinucleotides of $X_{0}$. Then, we prove our main result, i.e. a circular code is self-complementary if and only if the remaining two classes are complement of each other. Furthermore, we also show that a subset of $\mathcal{A}_{4}^{3} \backslash \mathcal{T}$ is a circular code if and only if the set consisting of all its complements is a circular code.
As a consequence of these results, we also prove that if a circular code is self-complementary then either both its two conjugated classes are circular codes or none is a circular code.

In Section 2, we give the necessary definitions and a characterization for a set of trinucleotides to be a circular code. In Section 3, we give the results, mainly expressed by Proposition 7 and Proposition 8 .

\section{Definitions}

The classical notions of alphabet, empty word, length, factor, proper factor, prefix, proper prefix, suffix, proper suffix, lexicographical order, etc. are those of [1]. Let $\mathcal{A}_{4}=\{A, C, G, T\}$ denote the genetic alphabet, lexicographically ordered with $A<C<G<T$. We use the following notation:

- $\mathcal{A}_{4}^{*}$ (respectively $\mathcal{A}_{4}^{+}$) is the set of words (respectively non-empty words) over $\mathcal{A}_{4}$;

- $\mathcal{A}_{4}^{2}$ is the set of the 16 words of length 2 (diletters or dinucleotides);

- $\mathcal{A}_{4}^{3}$ is the set of the 64 words of length 3 (triletters or trinucleotides).

We now recall two important genetic maps, the definitions of code and circular code, and the property of $C^{3}$ self-complementarity for a circular code, in particular $[1,6,17,24,25]$.

Definition 1. The complementarity map $\mathcal{C}: \mathcal{A}_{4}^{+} \rightarrow \mathcal{A}_{4}^{+}$ is defined by $\mathcal{C}(A)=T, \mathcal{C}(T)=A, \mathcal{C}(C)=G$ and $\mathcal{C}(G)=C$, and by $\mathcal{C}(u v)=\mathcal{C}(v) \mathcal{C}(u)$ for all $u, v \in \mathcal{A}_{4}^{+}$, e.g., $\mathcal{C}(A A C)=G T T$.

The map $\mathcal{C}$ on words is naturally extended to a word 
set $X$ : its complementary trinucleotide set $\mathcal{C}(X)$ is obtained by applying the complementarity map $\mathcal{C}$ to all the trinucleotides of $X$.

Definition 2. The circular permutation map $\mathcal{P}$ : $\mathcal{A}_{4}^{3} \rightarrow \mathcal{A}_{4}^{3}$ permutes circularly each trinucleotide $l_{1} l_{2} l_{3}$ as follows $\mathcal{P}\left(l_{1} l_{2} l_{3}\right)=l_{2} l_{3} l_{1}$.

The map $\mathcal{P}$ on words is also naturally extended to a word set $X$ : its permuted trinucleotide set $\mathcal{P}(X)$ is obtained by applying the circular permutation map $\mathcal{P}$ to all the trinucleotides of $X$. We shortly write $\mathcal{P}^{2}(X)$ for $\mathcal{P}(\mathcal{P}(X))$.

Definition 3. A set $X$ of words is a code if, for each $x_{1}, \cdots, x_{n}, x_{1}^{\prime}, \cdots, x_{m}^{\prime} \in X, n, m \geq 1$, the condition $x_{1} \cdots x_{n}$ $=x_{1}^{\prime} \cdots x_{m}^{\prime}$ implies $n=m$ and $x_{i}=x_{i}^{\prime}$ for $i=1, \cdots, n$.

Definition 4. A trinucleotide code $X$ is circular if, for each $x_{1}, \cdots, x_{n}, x_{1}^{\prime}, \cdots, x_{m}^{\prime} \in X, n, m \geq 1, p \in \mathcal{A}_{4}^{*}, \quad s \in \mathcal{A}_{4}^{+}$, the conditions $s x_{2} \cdots x_{n} p=x_{1}^{\prime} \cdots x_{m}^{\prime}$ and $x_{1}=p s$ imply $n=m, \quad p=\varepsilon \quad$ (empty word) and $x_{i}=x_{i}^{\prime}$ for $i=1, \cdots, n$.

Definition 5. A trinucleotide code $X$ is self-complementary if, for each $x \in X, \mathcal{C}(x) \in X$.

Definition 6. If $X_{0}$ is a subset of $\mathcal{A}_{4}^{3} \backslash \mathcal{T}$, we denote by $X_{1}$ the permuted trinucleotide set $\mathcal{P}\left(X_{0}\right)$ and by $X_{2}$ the permuted trinucleotide set $\mathcal{P}^{2}\left(X_{0}\right)$ and we call $X_{1}$ and $X_{2}$ the conjugated classes of $X_{0}$.

Definition 7. A trinucleotide circular code $X_{0}$ is $C^{3}$ self-complementary if $X_{0}, X_{1}$ and $X_{2}$ are circular codes satisfying the following properties: $X_{0}=\mathcal{C}\left(X_{0}\right)$ (self-complementary), $\mathcal{C}\left(X_{1}\right)=X_{2}$ (and $\left.\mathcal{C}\left(X_{2}\right)=X_{1}\right)$.

We have proved that there are exactly 528 self-complementary trinucleotide circular codes having 20 elements [6,24,25].

The concept of necklace was introduced by Pirillo [17] in order to characterize the circular codes for an efficient algorithm development. Let $l_{1}, l_{2}, \cdots, l_{n-1}, l_{n}, \cdots$ be letters in $\mathcal{A}_{4}, d_{1}, d_{2}, \cdots, d_{n-1}, d_{n}, \cdots$ diletters in $\mathcal{A}_{4}^{2}$ and $n \geq 2$ an integer.

Definition 8. Letter Diletter Continued Necklace (LDCN): We say that the ordered sequence $l_{1}, d_{1}, l_{2}, d_{2}, \cdots, d_{n-1}, l_{n}, d_{n}, l_{n+1}$ is an $(n+1) L D C N$ for $a$ subset $X \subset \mathcal{A}_{4}^{3}$ if

$$
l_{1} d_{1}, l_{2} d_{2}, \cdots, l_{n} d_{n} \in X
$$

and

$$
d_{1} l_{2}, d_{2} l_{3}, \cdots, d_{n-1} l_{n}, d_{n} l_{n+1} \in X .
$$

Any trinucleotide set is a code (more precisely, a uniform code [1]) but only few of them are circular codes. We have the following proposition.

Proposition 1. [17] Let $X$ be a trinucleotide code. The following conditions are equivalent:

1) $X$ is a circular code;

2) $X$ has no $5 L D C N$.
The figure below explains the notion of 5LDCN.

\begin{tabular}{lllllllllll}
$l_{1}$ & $d_{1}$ & $l_{2}$ & $d_{2}$ & $l_{3}$ & $d_{3}$ & $l_{4}$ & $d_{4}$ & $l_{5}$ \\
\hline
\end{tabular}

\section{Results}

Proposition 2. If $X_{0}$ is a trinucleotide circular code having 20 elements and $X_{1}$ and $X_{2}$ are its two conjugated classes then $X_{0}, X_{1}$ and $X_{2}$ constitute a partition of $\mathcal{A}_{4}^{3} \backslash \mathcal{T}$.

Proof. It is enough to prove that $X_{0} \cap X_{1}=X_{0} \cap X_{2}$ $=X_{1} \cap X_{2}=\varnothing$. Suppose that the trinucleotide $l_{1} l_{2} l_{3}$ belongs both to the classes $X_{0}$ and $X_{1}$. Then $l_{1} l_{2} l_{3}$ and $l_{3} l_{1} l_{2}$ are both in class $X_{0}$. As no two conjugated trinucleotides can belong to a circular code, we are in contradiction. Suppose that the trinucleotide $l_{1} l_{2} l_{3}$ belongs both to the classes $X_{0}$ and $X_{2}$. Then $l_{1} l_{2} l_{3}$ and $l_{2} l_{3} l_{1}$ are both in class $X_{0}$. As no two conjugated trinucleotides can belong to a circular code, we are in contradiction. Suppose that the trinucleotide $l_{1} l_{2} l_{3}$ belongs both to the classes $X_{1}$ and $X_{2}$. Then $l_{3} l_{1} l_{2}$ and $l_{2} l_{3} l_{1}$ are both in class $X_{0}$. As no two conjugated trinucleotides can belong to a circular code, we are in contradiction. So, $X_{0} \cap X_{1}=X_{0} \cap X_{2}=X_{1} \cap X_{2}=\varnothing$.

Proposition 3. The class of self-complementary circular codes $X_{0}$ with both $X_{1}$ and $X_{2}$ in the class of circular codes is non-empty.

Proof. Consider, for example, the following set $X_{0}$ of 20 trinucleotides

$$
\begin{aligned}
& X_{0}=\{A A C, A A G, A A T, A C C, A C G, A C T, \\
& A G C, A G G, A G T, A T C, A T T, C C T, C G T, \\
& C T T, G A T, G C C, G C T, G G C, G G T, G T T\} .
\end{aligned}
$$

It is enough to prove that $X_{0}$ is a self-complementary circular code and that its two conjugated classes $X_{1}$ and $X_{2}$ are also circular codes.

$X_{0}$ is a self-complementary circular code.

$X_{0}$ is self-complementary. Obvious by inspection.

$X_{0}$ is a circular code. We use Proposition 1 [17]. By way of contradiction, suppose that $X_{0}$ admits a $5 L D C N$. As $l_{2}$ can be $A, C, G$ or $T$, it is enough to prove that each choice leads to a contradiction.

1) If $l_{2}=A$ then there is no possible $d_{1}$ as $A$ is not a suffix of any trinucleotide of $X_{0}$, contradiction.

2) If $l_{2}=C$, there are three possible $d_{2}$ :

- if $d_{2}=C T$ (a) or $d_{2}=G T$ (b) then $l_{3}=T$ (c) but there is no possible $d_{3}$ as $T$ is not a prefix of any trinucleotide of $X_{0}$, contradiction,

- if $d_{2}=T T$ (d), there is a contradiction as no trinucleotide of $X_{0}$ has a prefix $T T$.

3) If $l_{2}=G$, there are six possible $d_{2}$ :

- if $d_{2}=C T$ or $d_{2}=G T$, contradiction (a) and (b),

- if $d_{2}=C C$ then $l_{3}=T$, contradiction (c),

- if $d_{2}=G C$ or $d_{2}=A T$ then $l_{3}=C$ or $l_{3}=T$ : 
- if $l_{3}=C$, there are three possible $d_{3}$ : if $d_{3}=C T$ or $d_{3}=G T$ then $l_{4}=T$, similarly to (c), contradiction, and if $d_{3}=T T$, similarly to (d), contradiction,

- if $l_{3}=T$, contradiction (c),

- if $d_{2}=T T$, contradiction (d).

4) If $l_{2}=T$, similarly to (c), contradiction.

As, for each letter, we cannot complete the assumed $5 L D C N$ for $X_{0}$, we are in contradiction. Hence, $X_{0}$ is a circular code.

$X_{1}=\mathcal{P}^{1}\left(X_{0}\right)$ is a circular code. We have to prove that

$$
\begin{aligned}
& X_{1}=\{\text { ACA, AGA, ATA, ATG, CCA, CCG, } \\
& C G A, C T A, C T C, C T G, G C A, G C G, G G A, \\
& \text { GTA, GTC, GTG, TCA,TTA,TTC,TTG }\}
\end{aligned}
$$

is a circular code. By way of contradiction, assume that $X_{1}$ admits a $5 L D C N$.

1) If $l_{2}=A$, there are four possible $d_{2}: C A, G A$, $T A$ and $T G$, but no possible $l_{3}$, contradiction.

2) If $l_{2}=C$, there are three possible $d_{1}: C T, G T$ and $T T$, but no possible $l_{1}$, contradiction.

3) If $l_{2}=G$, there are six possible $d_{1}: A T, C C$ and $G C$, and the cases $C T, G T$ and $T T$ already seen, but no possible $l_{1}$, contradiction.

4) If $l_{2}=T$, there is no possible $d_{1}$, contradiction.

Hence, $X_{1}$ is also a circular code.

$X_{2}=\mathcal{P}^{2}\left(X_{0}\right)$ is a circular code. Finally, we have to prove that

$$
\begin{aligned}
& X_{2}=\{C A A, C A C, C A G, C A T, C G C, C G G, \\
& G A A, G A C, G A G, T A A, T A C, T A G, T A T, \\
& T C C, T C G, T C T, T G A, T G C, T G G, T G T\}
\end{aligned}
$$

is a circular code. By way of contradiction, assume that $X_{2}$ admits a $5 L D C N$.

1) If $l_{2}=A$, there is no possible $d_{2}$, contradiction.

2) If $l_{2}=C$, there are six possible $d_{2}: A A, A C$, $A G, A T, G C$ and $G G$, but no possible $l_{3}$, contradiction.

3) If $l_{2}=G$, there are three possible $d_{2}: A A, A C$ and $A G$ which are cases already seen, contradiction.

4) If $l_{2}=T$, there are four possible $d_{1}: C A, T A$, $T C$ and $T G$, but no possible $l_{1}$, contradiction.

Hence, as $X_{0}$ and $X_{1}, X_{2}$ is also a circular code.

Proposition 4. The class of self-complementary circular codes $X_{0}$ having 20 elements with neither $X_{1}$ nor $\mathrm{X}_{2}$ in the class of circular codes is non-empty.

Proof. Consider, for example, the following set $X_{0}$ of 20 trinucleotides

$$
\begin{aligned}
& X_{0}=\{A A C, A A G, A A T, A C C, A C G, A C T, \\
& A G C, A G T, A T C, A T T, C G T, C T T, G A T, \\
& G C C, G C T, G G A, G G C, G G T, G T T, T C C\} .
\end{aligned}
$$

It is enough to prove that $X_{0}$ is a self-complementary circular code and that neither its conjugated class $X_{1}$ nor its conjugated class $X_{2}$ are circular codes.

$X_{0}$ is a self-complementary circular code.

$X_{0}$ is self-complementary. Obvious by inspection.

$X_{0}$ is a circular code. We use Proposition 1 [17]. By way of contradiction, assume that $X_{0}$ admits a $5 L D C N$.

1) If $l_{2}=A$ then there is one possible $d_{1}=G G$ but no possible $l_{1}$, contradiction.

2) If $l_{2}=C$, there are two possible $d_{2}$ :

- if $d_{2}=G T$ then $l_{3}=T$ (a) and $d_{3}=C C$ (b) but there is no possible $l_{4}$, contradiction,

- if $d_{2}=T T$ (c) then there is no possible $l_{3}$, contradiction.

3) If $l_{2}=G$ we have seven possible $d_{2}$ :

- if $d_{2}=A T$ then $l_{3}=C$ or $l_{3}=T$ :

- if $l_{3}=C$ (d) then $d_{3}=G T$ or $d_{3}=T T$ :

- if $d_{3}=G T$ then $l_{4}=T$ and $d_{4}=C C$ but there is no possible $l_{5}$, contradiction,

- if $d_{3}=T T$ then there is no possible $l_{4}$, contradiction,

- if $l_{3}=T$, contradiction (a),

- if $d_{2}=C C$, similarly to (b), contradiction,

- if $d_{2}=C T, d_{2}=G A$ or $d_{2}=G T$ then $l_{3}=T$, contradiction (a),

- if $d_{2}=G C$ then $l_{3}=C$ or $l_{3}=T$, contradiction (a) and (d),

- if $d_{2}=T T$, contradiction (c).

4) If $l_{2}=T$, similarly to (a), contradiction.

Hence, $X_{0}$ is a circular code.

$X_{1}=\mathcal{P}^{1}\left(X_{0}\right)$ is not a circular code. We have

$$
\begin{aligned}
& X_{1}=\{\text { ACA, AGA, ATA, ATG, CCA, CCG, } \\
& C C T, C G A, C T A, C T G, G A G, G C A, G C G, \\
& G T A, G T C, G T G, T C A, T T A, T T C, T T G\} .
\end{aligned}
$$

We use a technique developed in [23]. Observe that $X_{1}$ contains $\{A G A, C C T, G A G, T T C\}$. So,

$$
\begin{aligned}
& \left(l_{1}, d_{1}, l_{2}, d_{2}, l_{3}, d_{3}, l_{4}, d_{4}, l_{5}\right) \\
& =(A, G A, G, A G, A, G A, G, A G, A)
\end{aligned}
$$

is a $5 L D C N$ for this 4-element subset of $X_{1}$ and, a fortiori, for $X_{1}$ itself which, consequently, is not a circular code.

$$
\begin{aligned}
X_{2}= & \mathcal{P}^{2}\left(X_{0}\right) \text { is not a circular code. We have } \\
& X_{2}=\{A G G, C A A, C A C, C A G, C A T, C G C, \\
& C G G, C T C, G A A, G A C, T A A, T A C, T A G, \\
& T A T, T C G, T C T, T G A, T G C, T G G, T G T\} .
\end{aligned}
$$


We again use a technique developed in [23]. Remark that $X_{2}$ contains $\{G A A, C T C, A G G, T C T\}$. So,

$$
\begin{aligned}
& \left(l_{1}, d_{1}, l_{2}, d_{2}, l_{3}, d_{3}, l_{4}, d_{4}, l_{5}\right) \\
& =(T, C T, C, T C, T, C T, C, T C, T)
\end{aligned}
$$

is a $5 L D C N$ for this 4-element subset of $X_{2}$ and, a fortiori, for $X_{2}$ itself which, consequently, is not a circular code.

We need the propositions hereafter and, in particular the following one which states a general property of the involutional antiisomorphisms such as the complementary map $\mathcal{C}$.

Proposition 5. A subset $X$ of $\mathcal{A}_{4}^{3} \backslash \mathcal{T}$ is a circular code if and only if $\mathcal{C}(X)$ is a circular code.

Proof. Suppose, first, that $X$ is not a circular code and that $\mathcal{C}(X)$ is a circular code. So $X$ has a $5 L D C N$. This means that there are 13 nucleotides, say

$$
b_{1}, b_{2}, b_{3}, b_{4}, b_{5}, b_{6}, b_{7}, b_{8}, b_{9}, b_{10}, b_{11}, b_{12}, b_{13}
$$

such that the trinucleotides

$$
b_{1} b_{2} b_{3}, b_{4} b_{5} b_{6}, b_{7} b_{8} b_{9}, b_{10} b_{11} b_{12} \in X
$$

and

$$
b_{2} b_{3} b_{4}, b_{5} b_{6} b_{7}, b_{8} b_{9} b_{10}, b_{11} b_{12} b_{13} \in X .
$$

Now, consider the sequence

$$
\begin{aligned}
& \mathcal{C}\left(b_{13}\right), \mathcal{C}\left(b_{12}\right), \mathcal{C}\left(b_{11}\right), \mathcal{C}\left(b_{10}\right), \mathcal{C}\left(b_{9}\right), \mathcal{C}\left(b_{8}\right), \mathcal{C}\left(b_{7}\right), \\
& \mathcal{C}\left(b_{6}\right), \mathcal{C}\left(b_{5}\right), \mathcal{C}\left(b_{4}\right), \mathcal{C}\left(b_{3}\right), \mathcal{C}\left(b_{2}\right), \mathcal{C}\left(b_{1}\right) .
\end{aligned}
$$

All the following trinucleotides belong to $\mathcal{C}(X)$ :

$$
\begin{aligned}
& \mathcal{C}\left(b_{13}\right) \mathcal{C}\left(b_{12}\right) \mathcal{C}\left(b_{11}\right), \mathcal{C}\left(b_{10}\right) \mathcal{C}\left(b_{9}\right) \mathcal{C}\left(b_{8}\right), \\
& \mathcal{C}\left(b_{7}\right) \mathcal{C}\left(b_{6}\right) \mathcal{C}\left(b_{5}\right), \mathcal{C}\left(b_{4}\right) \mathcal{C}\left(b_{3}\right) \mathcal{C}\left(b_{2}\right) \in \mathcal{C}(X)
\end{aligned}
$$

and

$$
\begin{aligned}
& \mathcal{C}\left(b_{12}\right) \mathcal{C}\left(b_{11}\right) \mathcal{C}\left(b_{10}\right), \mathcal{C}\left(b_{9}\right) \mathcal{C}\left(b_{8}\right) \mathcal{C}\left(b_{7}\right), \\
& \mathcal{C}\left(b_{6}\right) \mathcal{C}\left(b_{5}\right) \mathcal{C}\left(b_{4}\right), \mathcal{C}\left(b_{3}\right) \mathcal{C}\left(b_{2}\right) \mathcal{C}\left(b_{1}\right) \in \mathcal{C}(X)
\end{aligned}
$$

as they are the complement of trinucleotides in $X$. So, $\mathcal{C}(X)$ admits a $5 L D C N$ and it cannot be a circular code. Contradiction.

The case $X$ is a circular code and $\mathcal{C}(X)$ is not a circular code is similar.

Proposition 6. Let $S$ be a self-complementary subset of $\mathcal{A}_{4}^{3} \backslash \mathcal{T}$. If $S$ is partitioned into three classes such that two of them are the complement of each other then necessarily the third one is self-complementary.

Proof. Let $X, Y$ and $Z$ be the three classes of an arbitrary partition of $S$ and suppose that $Y$ and $Z$ are complementary, i.e. $Y$ and $Z$ satisfy $\mathcal{C}(Y)=Z$. Let $t$ be a trinucleotide of $X$. We claim that $\mathcal{C}(t) \notin Y$. Indeed, in the opposite case, $Z$ should not be the complement of $Y$ because $t \in X$. We also claim that
$\mathcal{C}(t) \notin Z$. Indeed, in the opposite case, $Y$ should not be the complement of $Z$ because $t \in X$. It remains the case $\mathcal{C}(t) \in X$. So, $X$ is self-complementary.

Remark 1. Clearly, if $X, Y$ and $Z$ constitute an arbitrary partition of $\mathcal{A}_{4}^{3} \backslash \mathcal{T}$ then the self-complementarity of $X$ is not enough to ensure that $Y$ and $Z$ are complementary of each other. This remark is again true if, in addition, $X$ is a self-complementary circular code having 20 elements. Indeed in this case, it is easy to make a partition $\mathcal{A}_{4}^{3} \backslash\{X \cup \mathcal{T}\}$ in two classes $Y$ and $Z$ that are not complementary of each other. Any case, if we consider the partition of $\mathcal{A}_{4}^{3} \backslash \mathcal{T}$ in the three classes given by a self-complementary trinucleotide circular code $X_{0}$ having 20 elements and by its two conjugated classes $X_{1}$ and $X_{2}$ then the necessary and sufficient condition holds (Proposition 7 below).

Proposition 7. A trinucleotide circular code $X_{0}$ having 20 elements is self-complementary if and only if $X_{1}$ and $X_{2}$ are complement of each other.

Proof if part. It is a trivial consequence of Proposition 6.

Only if part. Suppose that $X_{0}$ is self-complementary and consider the partition $X_{0}, X_{1}$ and $X_{2}$ of $\mathcal{A}_{4}^{3} \backslash \mathcal{T}$. Suppose that the trinucleotide, say $l_{1} l_{2} l_{3}$, belongs to $X_{0}$. Then, also

$$
\mathcal{C}\left(l_{3}\right) \mathcal{C}\left(l_{2}\right) \mathcal{C}\left(l_{1}\right) \in X_{0} .
$$

We have

$$
l_{2} l_{3} l_{1}, \mathcal{C}\left(l_{2}\right) \mathcal{C}\left(l_{1}\right) \mathcal{C}\left(l_{3}\right) \in X_{1}
$$

and

$$
l_{3} l_{1} l_{2}, \mathcal{C}\left(l_{1}\right) \mathcal{C}\left(l_{3}\right) \mathcal{C}\left(l_{2}\right) \in X_{2} .
$$

As $l_{1} l_{2} l_{3}$ is a generic trinucleotide of $X_{0}$ and as $l_{2} l_{3} l_{1}$ is the complement of $\mathcal{C}\left(l_{1}\right) \mathcal{C}\left(l_{3}\right) \mathcal{C}\left(l_{2}\right)$

and

$$
\mathcal{C}\left(l_{2}\right) \mathcal{C}\left(l_{1}\right) \mathcal{C}\left(l_{3}\right) \text { is the complement of } l_{3} l_{1} l_{2}
$$

then $X_{1}$ is the complement of $X_{2}$.

As a consequence, we have the following proposition.

Proposition 8. If a trinucleotide circular code $X_{0}$ having 20 elements is self-complementary then either

1) $X_{1}$ and $X_{2}$ are both circular codes or

2) $X_{1}$ and $X_{2}$ are not circular codes (both have a necklace).

Proof. We have four possibilities:

$X_{1}$ is a circular code and $X_{2}$ is a circular code;

$X_{1}$ is a circular code and $X_{2}$ is not a circular code;

$X_{1}$ is not a circular code and $X_{2}$ is a circular code;

$X_{1}$ is not a circular code and $X_{2}$ is not a circular code.

Now, by applying Propositions 3 and 4, we have that 
the first and the last possibilities can be effectively realized.

Suppose that, by way of contradiction, the second possibility is realized. So, $X_{1}$ is a circular code. By Proposition 7, we have $\mathcal{C}\left(X_{1}\right)=X_{2}$. So, by Proposition 5, $X_{2}$ must also be a circular code. Contradiction.

Suppose that, by way of contradiction, the third possibility is realized. So, $X_{2}$ is a circular code. By Proposition 7, we have $\mathcal{C}\left(X_{2}\right)=X_{1}$. So, by Proposition 5, $X_{1}$ must also be a circular code. Contradiction.

So, only the first and the last possibilities can occur.

Hence, our proposition holds.

Proposition 9. The 528 self-complementary circular codes having 20 elements are partitioned into two classes: one class contains codes with the two permuted sets $X_{1}$ and $X_{2}$ which are both circular codes while the other class contains codes with the two permuted sets $X_{1}$ and $X_{2}$ which both are not circular codes.

Proof. It is enough to apply Proposition 8 to each of the 528 trinucleotide circular codes having 20 elements.

\section{Acknowledgements}

We thank Jacques Justin for his advices. The second author thanks the Dipartimento di matematica U. Dini for giving him a friendly hospitality.

\section{REFERENCES}

[1] J. Berstel and D. Perrin, "Theory of Codes, Vol. 117, (Pure and Applied Mathematics),” Academic Press, London, 1985.

[2] J.-L. Lassez, “Circular Codes and Synchronization,” International Journal of Computer \& Information Sciences, Vol. 5, 1976, pp. 201-208.

[3] F. H. C. Crick, J. S. Griffith and L. E. Orgel, "Codes without commas," Proceedings of the National Academy of Sciences of the USA, Vol. 43, 1957, pp. 416-421. doi:10.1073/pnas.43.5.416

[4] S. W. Golomb, B. Gordon and L. R. Welch, "CommaFree Codes," Canadian Journal of Mathematics, Vol. 10, No. 2, 1958, pp. 202-209. doi:10.4153/CJM-1958-023-9

[5] S. W. Golomb, L. R. Welch and M. Delbrück, "Construction and Properties of Comma-Free Codes," Biologiske Meddel Danske Vidensk Selsk, Vol. 23, 1958, pp. 1-34.

[6] D. G. Arquès and C. J. Michel, "A Complementary Circular Code in the Protein Coding Genes," Journal of Theoretical Biology, Vol. 182, No. 1, 1996, pp. 45-58. doi:10.1006/jtbi.1996.0142

[7] A. J. Koch and J. Lehman, "About a Symmetry of the Genetic Code,” Journal of Theoretical Biology, Vol. 189, No. 2, 1997, pp. 171-174. doi:10.1006/jtbi.1997.0503

[8] M.-P. Béal and J. Senellart, "On the Bound of the Synchronization Delay of a Local Automaton," Theoretical Computer Science, Vol. 205, No. 1-2, 1998, pp. 297-306. doi:10.1016/S0304-3975(98)80011-X
[9] F. Bassino, “Generating Function of Circular Codes,” Advances in Applied Mathematics, Vol. 22, No. 1, 1999, pp. 1-24. doi:10.1006/aama.1998.0613

[10] N. Štambuk, "On Circular Coding Properties of Gene and Protein Sequences,” Croatica Chemica Acta, Vol. 72, No. 4, 1999, pp. 999-1008.

[11] R. Jolivet and F. Rothen, "Peculiar Symmetry of DNA Sequences and Evidence Suggesting its Evolutionary Origin in a Primeval Genetic Code," First European Workshop Exo-/Astro-Biology, Noordwijk, 21-23 May 2001, pp. 173176.

[12] G. Frey and C. J. Michel, "Circular Codes in Archaeal Genomes,” Journal of Theoretical Biology, Vol. 223, No. 4, 2003, pp. 413-431. doi:10.1016/S0022-5193(03)00119-X

[13] C. Nikolaou and Y. Almirantis, "Mutually Symmetric and Complementary Triplets: Difference in their Use Distinguish Systematically between Coding and Non-Coding Genomic Sequences,” Journal of Theoretical Biology, Vol. 223, No. 4, 2003, pp. 477-487. doi:10.1016/S0022-5193(03)00123-1

[14] E. E. May, M. A. Vouk, D. L. Bitzer and D. I. Rosnick, "An Error-Correcting Framework for Genetic Sequence Analysis,” Journal of the Franklin Institute, Vol. 341, No. 1-2, 2004, pp. 89-109.

doi:10.1016/j.jfranklin.2003.12.009

[15] G. Frey and C. J. Michel, "Identification of Circular Codes in Bacterial Genomes and their Use in a Factorization Method for Retrieving the Reading Frames of Genes," Computational Biology and Chemistry, Vol. 30, No. 2, 2006, pp. 87-101. doi:10.1016/j.compbiolchem.2005.11.001

[16] J.-L. Lassez, R. A. Rossi and A. E. Bernal, “Crick’s Hypothesis Revisited: The Existence of a Universal Coding Frame," Proceedings of the 21st International Conference on Advanced Information Networking and Applications Workshops/Symposia (AINA'07), Niagara Falls, Vol. 2, 21-23 May 2007, pp. 745-751.

[17] G. Pirillo, “A characterization for a Set of Trinucleotides to be a Circular Code,” In: C. Pellegrini, P. Cerrai, P. Freguglia, V. Benci and G. Israel, Eds., Determinism, Holism, and Complexity, Kluwer, Boston, 2003.

[18] C. J. Michel, G. Pirillo and M. A. Pirillo, "Varieties of Comma-Free Codes," Computers \& Mathematics with Applications, Vol. 55, No. 5, 2008, pp. 989-996. doi:10.1016/j.camwa.2006.12.091

[19] G. Pirillo, “A Hierarchy for Circular Codes,” Theoretical Informatics and Applications, Vol. 42, No. 4, 2008, pp. 717-728.

[20] M. V. José, T. Govezensky, J. A. García and J. R. Bobadilla, "On the Evolution of the Standard Genetic Code: Vestiges of Critical Scale Invariance from the RNA World in Current Prokaryote Genomes," PLoS One, Vol. 4, No. 2, 2009, p. e4340. doi:10.1371/journal.pone.0004340

[21] G. Pirillo, "Some Remarks on Prefix and Suffix Codes," Pure Mathematics and Applications, Vol. 19, No. 2-3, 2008, pp. 53-60.

[22] G. Pirillo, “Non Sharing Border Codes,” Advances in 
Applied Mathematics, Vol. 3, No. 2, 2010, pp. 215-223.

[23] L. Bussoli, C. J. Michel and G. Pirillo, “On some Forbidden Configurations for Self-Complementary Trinucleotide Circular Codes,” Journal for Algebra Number Theory Academia, Vol. 2, 2011, pp. 223-232.

[24] G. Pirillo and M. A. Pirillo, "Growth Function of SelfComplementary Circular Codes,” Biology Forum, Vol. 98,
2005, pp. 97-110.

[25] C. J. Michel, G. Pirillo and M. A. Pirillo, “A Relation between Trinucleotide Comma-Free Codes and Trinucleotide Circular Codes,” Theoretical Computer Science, Vol. 401, No. 1-3, 2008, pp. 17-25. doi:10.1016/j.tcs.2008.02.049 